\title{
Some fixed-point results on (generalized) Bruck-Reilly $*$-extensions of monoids
}

\author{
Eylem Guzel Karpuz ${ }^{*}$, Ahmet Sinan Çevik², Jörg Koppitz ${ }^{3}$ and Ismail Naci Cangul ${ }^{4}$
}

\section{"Correspondence:}

eylem.guzel@kmu.edu.tr

'Department of Mathematics, Kamil

Özdag Science Faculty,

Karamanoglu Mehmetbey

University, Yunus Emre Campus,

Karaman, 70100, Turkey

Full list of author information is

available at the end of the article

\begin{abstract}
In this paper, we determine necessary and sufficient conditions for Bruck-Reilly and generalized Bruck-Reilly $*$-extensions of arbitrary monoids to be regular, coregular and strongly $\pi$-inverse. These semigroup classes have applications in various field of mathematics, such as matrix theory, discrete mathematics and $p$-adic analysis (especially in operator theory). In addition, while regularity and coregularity have so many applications in the meaning of boundaries (again in operator theory), inverse monoids and Bruck-Reilly extensions contain a mixture fixed-point results of algebra, topology and geometry within the purposes of this journal.
\end{abstract}

MSC: 20E22; 20M15; 20M18

Keywords: Bruck-Reilly extension; generalized Bruck-Reilly $*$-extension; $\pi$-inverse monoid; regular monoid

\section{Introduction and preliminaries}

In combinatorial group and semigroup theory, for a finitely generated semigroup (monoid), a fundamental question is to find its presentation with respect to some (irreducible) system of generators and relators, and then classify it with respect to semigroup classes. In this sense, in [1], the authors obtained a presentation for the Bruck-Reilly extension, which was studied previously by Bruck [2], Munn [3] and Reilly [4]. In different manners, this extension has been considered as a fundamental construction in the theory of semigroups. In detail, many classes of regular semigroups are characterized by BruckReilly extensions; for instance, any bisimple regular $w$-semigroup is isomorphic to a Reilly extension of a group [4] and any simple regular $w$-semigroup is isomorphic to a BruckReilly extension of a finite chain of groups [5, 6]. After that, in another important paper [7], the author obtained a new monoid, namely the generalized Bruck-Reilly *-extension, and presented the structure of the $*$-bisimple type $A w$-semigroup. Later on, in [8], the authors studied the structure theorem of the $*$-bisimple type $A w^{2}$-semigroups as the generalized Bruck-Reilly $*$-extension. Moreover, in a joint work [9], it has been recently defined a presentation for the generalized Bruck-Reilly $*$-extension and then obtained a Gröbner-Shirshov basis of this new construction. As we depicted in the abstract of this paper, Bruck-Reilly, its general version generalized Bruck-Reilly $*$-extension of monoids and semigroup classes are not only important in combinatorial algebra but also in linear algebra, discrete mathematics and topology. So these semigroup classes, regular, coregular, inverse and strongly $\pi$-inverse, are the most studied classes in algebra.

(c) 2013 Guzel Karpuz et al.: licensee Springer. This is an Open Access article distributed under the terms of the Creative Commons Attribution License (http://creativecommons.org/licenses/by/2.0), which permits unrestricted use, distribution, and reproduction in any medium, provided the original work is properly cited. 
In this paper, as a next step of these above results, we investigate regularity, coregularity and strongly $\pi$-inverse properties over Bruck-Reilly and generalized Bruck-Reilly $*$-extensions of monoids. We recall that regularity and strongly $\pi$-inverse properties have been already studied for some other special extensions (semidirect and wreath products) of monoids [10,11]. We further recall that these two important properties have been also investigated for the semidirect product version of Schützenberger products of any two monoids $[12,13]$. However, there are not yet such investigations concerning coregularity. As we depicted in the abstract, semigroup classes have important applications in various fields of mathematics, such as matrix theory, discrete mathematics and $p$-adic analysis (especially in operator theory). In addition, while regularity and coregularity have so many applications in the meaning of boundaries (again in operator theory), inverse monoids and Bruck-Reilly extensions contain a mixture of algebra, topology and geometry within the purposes of this journal.

Now let us present the following fundamental material that will be needed in this paper. We refer the reader to [14-16] for more detailed knowledge.

An element $a$ of a semigroup $S$ is called regular if there exists $x \in S$ such that $a x a=a$. The semigroup $S$ is called regular if all its elements are regular. Groups are of course regular semigroups, but the class of regular semigroups is vastly more extensive than the class of groups (see [16]). Further, to have an inverse element can also be important in a semigroup. Therefore, we call $S$ is an inverse semigroup if every element has exactly one inverse. The well-known examples of inverse semigroups are groups and semilattices. An element $a \in S$ is called coregular and $b$ its coinverse if $a=a b a=b a b$. A semigroup $S$ is said to be coregular if each element of $S$ is coregular [17]. In addition, let $E(S)$ and $\operatorname{Reg} S$ be the set of idempotent and regular elements, respectively. We then say that $S$ is called $\pi$-regular if, for every $s \in S$, there is an $m \in \mathbb{N}$ such that $s^{m} \in \operatorname{Reg} S$. Moreover, if $S$ is $\pi$-regular and the set $E(S)$ is a commutative subsemigroup of $S$, then $S$ is called strongly $\pi$-inverse semigroup [16]. We recall that $\operatorname{Reg} S$ is an inverse subsemigroup of a strongly $\pi$-inverse semigroup $S$.

\section{Bruck-Reilly extensions of monoids}

Let us suppose that $A$ is a monoid with an endomorphism $\theta$ defined on it such that $A \theta$ is in the $\mathcal{H}$-class [16] of the identity $1_{A}$ of $A$. Also, let $\mathbb{N}^{0}$ denotes the set of nonnegative integers. Hence, the set $\mathbb{N}^{0} \times A \times \mathbb{N}^{0}$ with the multiplication

$$
(m, a, n)\left(m^{\prime}, a^{\prime}, n^{\prime}\right)=\left(m-n+t,\left(a \theta^{t-n}\right)\left(a^{\prime} \theta^{t-m^{\prime}}\right), n^{\prime}-m^{\prime}+t\right),
$$

where $t=\max \left(n, m^{\prime}\right)$ and $\theta^{0}$ is the identity map on $A$, forms a monoid with identity $\left(0,1_{A}, 0\right)$. Then this monoid is called the Bruck-Reilly extension of $A$ determined by $\theta$ [2-4] and denoted by $B R(A, \theta)$.

In the above references, the authors used $B R(A, \theta)$ to prove that every semigroup embeds in a simple monoid, and to characterize special classes of inverse semigroups. In [3, Theorem 3.1], Munn showed that $B R(A, \theta)$ is an inverse semigroup if and only if $A$ is inverse. So, the following result is a direct consequence of this theorem.

Corollary 1 Let $A$ be an arbitrary monoid. Then $B R(A, \theta)$ is regular if and only if $A$ is regular. 
For $m, m^{\prime} \in \mathbb{N}^{0}$ and $a, a^{\prime} \in A$, since

$$
(m, a, m)\left(m^{\prime}, a^{\prime}, m^{\prime}\right)=\left(m-m+t,\left(a \theta^{t-m}\right)\left(a^{\prime} \theta^{t-m^{\prime}}\right), m^{\prime}-m^{\prime}+t\right)=(t, b, t)
$$

with $t=\max \left(m, m^{\prime}\right)$ and $b=\left(a \theta^{t-m}\right)\left(a^{\prime} \theta^{t-m^{\prime}}\right) \in A$, the set $\left\{(m, a, m) \mid a \in A, m \in \mathbb{N}^{0}\right\}$ becomes a subsemigroup of $B R(A, \theta)$. Thus, we further have the following lemma.

Lemma 1 Let $(m, a, n) \in B R(A, \theta)$. If $(m, a, n)$ is coregular then $m=n$.

Proof Let $(m, a, n) \in B R(A, \theta)$. Then there exists $\left(m^{\prime}, a^{\prime}, n^{\prime}\right) \in B R(A, \theta)$ such that

$$
(m, a, n)\left(m^{\prime}, a^{\prime}, n^{\prime}\right)(m, a, n)=(m, a, n) \quad \text { and } \quad\left(m^{\prime}, a^{\prime}, n^{\prime}\right)(m, a, n)\left(m^{\prime}, a^{\prime}, n^{\prime}\right)=(m, a, n)
$$

We have

$$
(m, a, n)\left(m^{\prime}, a^{\prime}, n^{\prime}\right)(m, a, n)=\left(m-n-n^{\prime}+m^{\prime}+s^{\prime}, b, n-m+s^{\prime}\right)
$$

for some $b \in A$, where $s=\max \left(n, m^{\prime}\right)$ and $s^{\prime}=\max \left(n^{\prime}-m^{\prime}+s, m\right)$. This implies $m=m-n-$ $n^{\prime}+m^{\prime}+s^{\prime}$ and $n=n-m+s^{\prime}$, in other words $m+m^{\prime}=n+n^{\prime}$. Further, for some $c \in A$, we have

$$
\left(m^{\prime}, a^{\prime}, n^{\prime}\right)(m, a, n)\left(m^{\prime}, a^{\prime}, n^{\prime}\right)=\left(m^{\prime}-n^{\prime}-n+m+S^{\prime}, c, n^{\prime}-m^{\prime}+S^{\prime}\right),
$$

where $S=\max \left(n^{\prime}, m\right)$ and $S^{\prime}=\max \left(n-m+S, m^{\prime}\right)$. This gives $m=m^{\prime}-n^{\prime}-n+m+S^{\prime}$, $n=n^{\prime}-m^{\prime}+S^{\prime}$, and consequently, $n^{\prime}=m^{\prime}$. Together with $m+m^{\prime}=n+n^{\prime}$, we obtain $m=n$ as required.

Lemma 1 shows that a coregular element in $B R(A, \theta)$ and its coinverse belongs to

$$
\left\{(m, a, m) \mid a \in A, m \in \mathbb{N}^{0}\right\} .
$$

Now we can present the following result.

Theorem 1 Let $A$ be a monoid. Then $A^{\prime}=\left\{(m, a, m) \mid a \in A, m \in \mathbb{N}^{0}\right\} \leq B R(A, \theta)$ is coregular if and only if $A$ is coregular.

Proof Assume that $A^{\prime} \leq B R(A, \theta)$ is a coregular monoid. For $(0, a, 0) \in B R(A, \theta)$, there exists $\left(m^{\prime}, a^{\prime}, m^{\prime}\right) \in B R(A, \theta)$ such that

$$
(0, a, 0)\left(m^{\prime}, a^{\prime}, m^{\prime}\right)(0, a, 0)=\left(m^{\prime},\left(a \theta^{m^{\prime}}\right) a^{\prime}\left(a \theta^{m^{\prime}}\right), m^{\prime}\right)=(0, a, 0)
$$

and

$$
\left(m^{\prime}, a^{\prime}, m^{\prime}\right)(0, a, 0)\left(m^{\prime}, a^{\prime}, m^{\prime}\right)=\left(m^{\prime}, a^{\prime}\left(a \theta^{m^{\prime}}\right) a^{\prime}, m^{\prime}\right)=(0, a, 0)
$$

By (1) and (2), we clearly have $m^{\prime}=0$, and hence, $a a^{\prime} a=a$ and $a^{\prime} a a^{\prime}=a$. So $A$ is coregular. 
Conversely, let $(m, a, m) \in B R(A, \theta)$. Then there is an $a^{\prime} \in A$ with $a a^{\prime} a=a$ and $a^{\prime} a a^{\prime}=a$. Thus, for $\left(m, a^{\prime}, m\right) \in B R(A, \theta)$, we get

$$
(m, a, m)\left(m, a^{\prime}, m\right)(m, a, m)=\left(m, a a^{\prime} a, m\right)=(m, a, m)
$$

and

$$
\left(m, a^{\prime}, m\right)(m, a, m)\left(m, a^{\prime}, m\right)=\left(m, a^{\prime} a a^{\prime}, m\right)=(m, a, m) .
$$

Therefore, $A^{\prime}=\left\{(m, a, m) \mid a \in A, m \in \mathbb{N}^{0}\right\} \leq B R(A, \theta)$ is coregular.

In [3, Theorem 3.1], it is proved that:

- $(m, a, n)$ is an idempotent element in $B R(A, \theta)$ if and only if $m=n$ and $a$ is an idempotent element in $A$.

This result will be used in the proof of the following theorem.

Theorem $2 B R(A, \theta)$ is strongly $\pi$-inverse if and only if $A$ is regular and the idempotents in A commute.

Proof Let $B R(A, \theta)$ be strongly $\pi$-inverse, and let $a \in A$. Also let us consider the element $(0, a, 1)$ in $B R(A, \theta)$. Then there exists an element $r \in \mathbb{N}$ with $(0, a, 1)^{r} \in \operatorname{Reg} B R(A, \theta)$. It is actually a routine matter to show that $(0, a, 1)^{r}=\left(0, a(a \theta)^{r-1}, r\right)$. Moreover, there exists an element $a^{\prime} \in A$ such that $\left(r, a^{\prime}, 0\right)$ is an inverse of $\left(0, a(a \theta)^{r-1}, r\right)$ (see [3]). Therefore,

$$
\begin{aligned}
\left(0, a(a \theta)^{r-1}, r\right) & =\left(0, a(a \theta)^{r-1}, r\right)\left(r, a^{\prime}, 0\right)\left(0, a(a \theta)^{r-1}, r\right) \\
& =\left(0, a(a \theta)^{r-1} a^{\prime}, r\right)\left(0, a(a \theta)^{r-1}, r\right)=\left(0, a(a \theta)^{r-1} a^{\prime} a(a \theta)^{r-1}, r\right) .
\end{aligned}
$$

This shows that

$$
a(a \theta)^{r-1}=a(a \theta)^{r-1} a^{\prime} a(a \theta)^{r-1} .
$$

By the assumption given in the beginning of this section, since $a \theta$ is in the $\mathcal{H}$-class of the element $1_{A}$, we obtain $a \theta$ is a group element, and so there is an inverse element $\left((a \theta)^{r-1}\right)^{-1}$. Thus, by (3), we get $a=a(a \theta)^{r-1} a^{\prime} a$; in other words, $a \in \operatorname{Reg} A$. Consequently, $A$ is regular. Now, let us also show that the elements in $E(A)$ are commutative. But this is quite clear by the fact that the idempotents in $B R(A, \theta)$ commute if and only if the idempotents in $A$ commute (see [3, Theorem 3.1(5)]).

Conversely, let us suppose that $A$ is regular and the idempotents in $A$ commute. Then $B R(A, \theta)$ is regular, where $\pi$-regular by Corollary 1. Moreover, again by [3, Theorem 3.1(5)], $E(B R(A, \theta))$ is a commutative subsemigroup, which is required to $B R(A, \theta)$ satisfy strongly $\pi$-inverse property, hence the result.

\section{The generalized Bruck-Reilly $*$-extension of monoids}

Suppose that $A$ is an arbitrary monoid having $H_{1}^{*}$ and $H_{1}$ as the $\mathcal{H}^{*}$ - and $\mathcal{H}$ - classes containing the identity element $1_{A}$ of $A$. Moreover, let us assume that $\beta$ and $\gamma$ are morphisms 
from $A$ into $H_{1}^{*}$ and, for an element $u$ in $H_{1}$, let $\lambda_{u}$ be the inner automorphism of $H_{1}^{*}$ defined by $x \mapsto u x u^{-1}$ such that $\gamma \lambda_{u}=\beta \gamma$.

Now one can consider the set $S=\mathbb{N}^{0} \times \mathbb{N}^{0} \times A \times \mathbb{N}^{0} \times \mathbb{N}^{0}$ into a semigroup with a multiplication

$$
\begin{aligned}
& (m, n, v, p, q)\left(m^{\prime}, n^{\prime}, v^{\prime}, p^{\prime}, q^{\prime}\right) \\
& \quad= \begin{cases}\left(m, n-p+d,\left(v \beta^{d-p}\right)\left(v^{\prime} \beta^{d-n^{\prime}}\right), p^{\prime}-n^{\prime}+d, q^{\prime}\right), & \text { if } q=m^{\prime}, \\
\left.\left(m, n, v\left(\left(u^{-n^{\prime}}\left(v^{\prime} \gamma\right) u^{p^{\prime}}\right) \gamma^{q-m^{\prime}-1}\right) \beta^{p}\right), p, q^{\prime}-m^{\prime}+q\right), & \text { if } q>m^{\prime}, \\
\left(m-q+m^{\prime}, n^{\prime},\left(\left(\left(u^{-n}(v \gamma) u^{p}\right) \gamma^{m^{\prime}-q-1}\right) \beta^{n^{\prime}}\right) v^{\prime}, p^{\prime}, q^{\prime}\right), & \text { if } q<m^{\prime},\end{cases}
\end{aligned}
$$

where $d=\max \left(p, n^{\prime}\right)$ and $\beta^{0}, \gamma^{0}$ are interpreted as the identity map of $A$, and also $u^{0}$ is interpreted as the identity $1_{A}$ of $A$. In [8], Yu Shung and Li-Min Wang showed that $S$ is a monoid with the identity $\left(0,0,1_{A}, 0,0\right)$. In fact, this new monoid $S=\mathbb{N}^{0} \times \mathbb{N}^{0} \times A \times$ $\mathbb{N}^{0} \times \mathbb{N}^{0}$ is denoted by $G B R^{*}(A ; \beta, \gamma ; u)$ and called generalized Bruck-Reilly $*$-extension of $A$ determined by the morphisms $\beta, \gamma$ and the element $u$.

The following lemmas were established in [8].

Lemma 2 If $(m, n, v, p, q) \in G B R^{*}(A ; \beta, \gamma ; u)$, then $(m, n, v, p, q)$ is an idempotent if and only if $m=q, n=p$ and $v$ is idempotent.

Lemma 3 If $(m, n, v, p, q) \in G B R^{*}(A ; \beta, \gamma ; u)$, then $(m, n, v, p, q)$ has an inverse

$$
\left(m^{\prime}, n^{\prime}, v^{\prime}, p^{\prime}, q^{\prime}\right) \in S
$$

if and only if $v^{\prime}$ is an inverse of $v$ in $A$ while $m^{\prime}=q, n^{\prime}=p, p^{\prime}=n$ and $q^{\prime}=m$.

Then we have an immediate consequence as in the following.

Corollary 2 Let $A$ be a monoid. Then $G_{B R}^{*}(A ; \beta, \gamma ; u)$ is regular if and only if $A$ is regular.

In this section, we mainly characterize the properties coregularity and strongly $\pi$ inverse over the generalized Bruck-Reilly $*$-extensions of monoids. More specifically, for a given monoid $A$, we determine the maximal submonoid of $G B R^{*}(A ; \beta, \gamma ; u)$, which can be held coregularity if $A$ satisfies particular properties.

Our first observation is the following.

Lemma 4 The set $\mathcal{L}:=\left\{(m, n, v, n, m) \mid v \in A, m, n \in \mathbb{N}^{0}\right\}$ is a submonoid of $G B R^{*}(A ; \beta, \gamma ; u)$.

Proof By considering the multiplication in (4), the proof can be seen easily.

It turns out that all coregular elements in $G B R^{*}(A ; \beta, \gamma ; u)$ belong to the submonoid $\mathcal{L}$.

Lemma 5 Let $(m, n, v, p, q) \in G B R^{*}(A ; \beta, \gamma ; u)$. If $(m, n, v, p, q)$ is coregular then $m=q$ and $n=p$. 
Proof Let $(m, n, v, p, q) \in G B R^{*}(A ; \beta, \gamma ; u)$ be a coregular element. Then there exists an element $\left(m^{\prime}, n^{\prime}, v^{\prime}, p^{\prime}, q^{\prime}\right) \in G B R^{*}(A ; \beta, \gamma ; u)$ such that

$$
\begin{aligned}
& (m, n, v, p, q)\left(m^{\prime}, n^{\prime}, v^{\prime}, p^{\prime}, q^{\prime}\right)(m, n, v, p, q)=(m, n, v, p, q) \quad \text { and } \\
& \left(m^{\prime}, n^{\prime}, v^{\prime}, p^{\prime}, q^{\prime}\right)(m, n, v, p, q)\left(m^{\prime}, n^{\prime}, v^{\prime}, p^{\prime}, q^{\prime}\right)=(m, n, v, p, q) .
\end{aligned}
$$

Let $q=m^{\prime}$ and $q^{\prime}=m$. Then we have

$$
\begin{aligned}
& (m, n, v, p, q)\left(m^{\prime}, n^{\prime}, v^{\prime}, p^{\prime}, q^{\prime}\right)(m, n, v, p, q) \\
& \quad=\left(m, n+n^{\prime}-p-p^{\prime}+z^{\prime}, w, p-n+z^{\prime}, q\right)
\end{aligned}
$$

for some $w \in A$, where $z=\max \left(p, n^{\prime}\right)$ and $z^{\prime}=\max \left(p^{\prime}-n^{\prime}+z, n\right)$. This implies that

$$
n=n+n^{\prime}-p-p^{\prime}+z^{\prime}
$$

and

$$
p=p-n+z^{\prime}
$$

By (6), we have $n=z^{\prime}$. Applying this in (5), we get $n+n^{\prime}-p-p^{\prime}+n=n$ and thus $n+n^{\prime}=p+p^{\prime}$. Further, we have

$$
\begin{aligned}
& \left(m^{\prime}, n^{\prime}, v^{\prime}, p^{\prime}, q^{\prime}\right)(m, n, v, p, q)\left(m^{\prime}, n^{\prime}, v^{\prime}, p^{\prime}, q^{\prime}\right) \\
& =\left(m^{\prime}, n^{\prime}+n-p^{\prime}-p+Z^{\prime}, w^{\prime}, p^{\prime}-n^{\prime}+Z^{\prime}, q^{\prime}\right)
\end{aligned}
$$

for some $w^{\prime} \in A$, where $Z=\max \left(p^{\prime}, n\right)$ and $Z^{\prime}=\max \left(p-n+Z, n^{\prime}\right)$. This implies that $m=m^{\prime}$, $q=q^{\prime}$,

$$
n=n^{\prime}+n-p^{\prime}-p+Z^{\prime}
$$

and

$$
p=p^{\prime}-n^{\prime}+Z^{\prime}
$$

By writing the equality (8) in (7), we get $n^{\prime}=p^{\prime}$. Together with $n+n^{\prime}=p+p^{\prime}$, we obtain $n=p$. By assuming $q=m^{\prime}$, we also get $m=q$.

Now let $(x, y, t, z, w)\left(x^{\prime}, y^{\prime}, t^{\prime}, z^{\prime}, w^{\prime}\right)=\left(x^{\prime \prime}, y^{\prime \prime}, t^{\prime \prime}, z^{\prime \prime}, w^{\prime \prime}\right)$. Then it is easy to verify that $x^{\prime \prime} \geq$ $x^{\prime}, x$ and $y^{\prime \prime} \geq y^{\prime}, y$. If $w \neq x^{\prime}$, we can easily see that $x^{\prime \prime}>x^{\prime}, x$ or $y^{\prime \prime}>y^{\prime}, y$. This shows that $(m, n, a, p, q)\left(m^{\prime}, n^{\prime}, a^{\prime}, p^{\prime}, q^{\prime}\right)(m, n, a, p, q) \neq(m, n, a, p, q)$ if $q \neq m^{\prime}$ or $q^{\prime} \neq m$. Hence, $q \neq m^{\prime}$ or $q^{\prime} \neq m$ is not possible.

Then we have the following result.

Theorem 3 Let $A$ be a monoid. Then the submonoid $\mathcal{L}$ of $G B R^{*}(A ; \beta, \gamma ; u)$ is coregular if and only if $A$ is coregular. 
Proof Suppose that $\mathcal{L}=\left\{(m, n, v, n, m) \mid v \in A, m, n \in \mathbb{N}^{0}\right\} \leq G B R^{*}(A ; \beta, \gamma ; u)$ is coregular. For each $\left(m^{\prime}, 0, v, 0, m^{\prime}\right)$ in $\mathcal{L}$, there exists an element $\left(m^{\prime}, n^{\prime}, v^{\prime}, n^{\prime}, m^{\prime}\right) \in \mathcal{L}$ such that

$$
\begin{aligned}
\left(m^{\prime}, 0, v, 0, m^{\prime}\right)\left(m^{\prime}, n^{\prime}, v^{\prime}, n^{\prime}, m^{\prime}\right)\left(m^{\prime}, 0, v, 0, m^{\prime}\right) & =\left(m^{\prime}, n^{\prime},\left(v \beta^{n^{\prime}}\right) v^{\prime}\left(v \beta^{n^{\prime}}\right), n^{\prime}, m^{\prime}\right) \\
& =\left(m^{\prime}, 0, v, 0, m^{\prime}\right)
\end{aligned}
$$

and

$$
\begin{aligned}
\left(m^{\prime}, n^{\prime}, v^{\prime}, n^{\prime}, m^{\prime}\right)\left(m^{\prime}, 0, v, 0, m^{\prime}\right)\left(m^{\prime}, n^{\prime}, v^{\prime}, n^{\prime}, m^{\prime}\right) & =\left(m^{\prime}, n^{\prime}, v^{\prime}\left(v \beta^{n^{\prime}}\right) v^{\prime}, n^{\prime}, m^{\prime}\right) \\
& =\left(m^{\prime}, 0, v, 0, m^{\prime}\right) .
\end{aligned}
$$

By (9) and (10), we obtain $n^{\prime}=0$, and hence $v v^{\prime} v=v$ and $v^{\prime} v v^{\prime}=v$. So, $A$ is coregular.

Conversely, let $A$ be a coregular monoid and $(m, n, v, n, m) \in \mathcal{L}$. Then there exists an element $v^{\prime} \in A$ with $v v^{\prime} v=v$ and $v^{\prime} v v^{\prime}=v$. Therefore, for $\left(m, n, v^{\prime}, n, m\right) \in \mathcal{L}$, we get

$$
\begin{aligned}
& (m, n, v, n, m)\left(m, n, v^{\prime}, n, m\right)(m, n, v, n, m)=\left(m, n, v v^{\prime} v, n, m\right)=(m, n, v, n, m), \\
& \left(m, n, v^{\prime}, n, m\right)(m, n, v, n, m)\left(m, n, v^{\prime}, n, m\right)=\left(m, n, v^{\prime} v v^{\prime}, n, m\right)=(m, n, v, n, m) .
\end{aligned}
$$

Hence, $\mathcal{L} \leq G B R^{*}(A ; \beta, \gamma ; u)$ is a coregular monoid, as desired.

In the final theorem, we consider strongly $\pi$-inverse property.

Theorem $4 G B R^{*}(A ; \beta, \gamma ; u)$ is strongly $\pi$-inverse if and only if $A$ is regular and the idempotents in A commute.

Proof We will follow the same format as in the proof of Theorem 2. So, let us suppose that $G B R^{*}(A ; \beta, \gamma ; u)$ is a strongly $\pi$-inverse monoid, and let $a \in A$. Then, for $(0,0, a, 1,0) \in$ $G B R^{*}(A ; \beta, \gamma ; u)$, there is an element $r \in \mathbb{N}$ with $(0,0, a, 1,0)^{r} \in \operatorname{Reg} G B R^{*}(A ; \beta, \gamma ; u)$. It is easily seen that $(0,0, a, 1,0)^{r}=\left(0,0, a(a \beta)^{r-1}, r, 0\right)$. Moreover, there is an element $a^{\prime} \in A$ such that $\left(0, r, a^{\prime}, 0,0\right)$ is an inverse of $\left(0,0, a(a \beta)^{r-1}, r, 0\right)$ by Lemma 3 . From here, we have

$$
\begin{aligned}
\left(0,0, a(a \beta)^{r-1}, r, 0\right) & =\left(0,0, a(a \beta)^{r-1}, r, 0\right)\left(0, r, a^{\prime}, 0,0\right)\left(0,0, a(a \beta)^{r-1}, r, 0\right) \\
& =\left(0,0, a(a \beta)^{r-1} a^{\prime} a(a \beta)^{r-1}, r, 0\right) .
\end{aligned}
$$

This actually shows that

$$
a(a \beta)^{r-1}=a(a \beta)^{r-1} a^{\prime} a(a \beta)^{r-1} .
$$

At the same time, since $a \beta$ is in the $\mathcal{H}^{*}$-class of the $1_{A}$, there exists an inverse element $\left((a \beta)^{r-1}\right)^{-1}$. Thus, by (11), we get $a=a(a \beta)^{r-1} a^{\prime} a$, in other words, $a \in \operatorname{Reg} A$. Hence, $A$ is regular. Now, let us show that the elements in $E(A)$ are commutative to conclude the necessity part of the proof. To do that, consider any two elements $v_{1}$ and $v_{2}$ in $E(A)$. Thus, $\left(0,0, v_{1}, 0,0\right),\left(0,0, v_{2}, 0,0\right) \in E\left(G B R^{*}(A ; \beta, \gamma ; u)\right)$ (by Lemma 2$)$ and we have

$$
\begin{aligned}
\left(0,0, v_{1} v_{2}, 0,0\right) & =\left(0,0, v_{1}, 0,0\right)\left(0,0, v_{2}, 0,0\right) \\
& =\left(0,0, v_{2}, 0,0\right)\left(0,0, v_{1}, 0,0\right)=\left(0,0, v_{2} v_{1}, 0,0\right) .
\end{aligned}
$$

So, $v_{1} v_{2}=v_{2} v_{1}$, as required. 
Conversely, let us suppose that $A$ is regular. Then $\operatorname{GBR}^{*}(A ; \beta, \gamma ; u)$ is regular, where $\pi$ regular by Corollary 2. Now we need to show that the elements in $E\left(G B R^{*}(A ; \beta, \gamma ; u)\right)$ commute. To do that, let us take $(m, n, e, n, m),\left(m^{\prime}, n^{\prime}, e^{\prime}, n^{\prime}, m^{\prime}\right) \in E\left(G B R^{*}(A ; \beta, \gamma ; u)\right)$, and thus $e e^{\prime}=e^{\prime} e$ by Lemma 2. Now, by considering the multiplication $(m, n, e, n, m)\left(m^{\prime}, n^{\prime}, e^{\prime}, n^{\prime}, m^{\prime}\right)$ as defined in (4), we have the following cases.

Case (i): If $m=m^{\prime}$, then we get

$$
(m, n, e, n, m)\left(m^{\prime}, n^{\prime}, e^{\prime}, n^{\prime}, m^{\prime}\right)=\left(m, d^{\prime},\left(e \beta^{d^{\prime}-n}\right)\left(e^{\prime} \beta^{d^{\prime}-n^{\prime}}\right), d^{\prime}, m^{\prime}\right)
$$

and

$$
\left(m^{\prime}, n^{\prime}, e^{\prime}, n^{\prime}, m^{\prime}\right)(m, n, e, n, m)=\left(m^{\prime}, d^{\prime},\left(e^{\prime} \beta^{d^{\prime}-n^{\prime}}\right)\left(e \beta^{d^{\prime}-n}\right), d^{\prime}, m^{\prime}\right)
$$

respectively, where $d^{\prime}=\max \left(n, n^{\prime}\right)$. Since $e, e^{\prime} \in E(A)$, we deduce that both $e^{\prime} \beta^{d^{\prime}-n^{\prime}}$ and $e \beta^{d^{\prime}-n}$ are the elements of $E(A)$, in other words,

$$
\left(e^{\prime} \beta^{d^{\prime}-n^{\prime}}\right)\left(e \beta^{d^{\prime}-n}\right)=\left(e \beta^{d^{\prime}-n}\right)\left(e^{\prime} \beta^{d^{\prime}-n^{\prime}}\right)
$$

Thus, $(m, n, e, n, m)\left(m^{\prime}, n^{\prime}, e^{\prime}, n^{\prime}, m^{\prime}\right)=\left(m^{\prime}, n^{\prime}, e^{\prime}, n^{\prime}, m^{\prime}\right)(m, n, e, n, m)$.

Case (ii): If $m<m^{\prime}$ or $m>m^{\prime}$, then we get

$$
\begin{aligned}
& (m, n, e, n, m)\left(m^{\prime}, n^{\prime}, e^{\prime}, n^{\prime}, m^{\prime}\right)=\left(m^{\prime}, n^{\prime},\left(\left(\left(u^{-n}(e \gamma) u^{n}\right) \gamma^{m^{\prime}-m-1}\right) \beta^{n^{\prime}}\right) e^{\prime}, n^{\prime}, m^{\prime}\right), \\
& \left(m^{\prime}, n^{\prime}, e^{\prime}, n^{\prime}, m^{\prime}\right)(m, n, e, n, m)=\left(m^{\prime}, n^{\prime}, e^{\prime}\left(\left(\left(u^{-n}(e \gamma) u^{n}\right) \gamma^{m^{\prime}-m-1}\right) \beta^{n^{\prime}}\right), n^{\prime}, m^{\prime}\right)
\end{aligned}
$$

or

$$
\begin{aligned}
& (m, n, e, n, m)\left(m^{\prime}, n^{\prime}, e^{\prime}, n^{\prime}, m^{\prime}\right)=\left(m, n,\left(\left(\left(u^{-n^{\prime}}\left(e^{\prime} \gamma\right) u^{n^{\prime}}\right) \gamma^{m-m^{\prime}-1}\right) \beta^{n}\right) e^{\prime}, n, m\right), \\
& \left(m^{\prime}, n^{\prime}, e^{\prime}, n^{\prime}, m^{\prime}\right)(m, n, e, n, m)=\left(m, n, e^{\prime}\left(\left(\left(u^{-n^{\prime}}\left(e^{\prime} \gamma\right) u^{n^{\prime}}\right) \gamma^{m-m^{\prime}-1}\right) \beta^{n}\right), n, m\right),
\end{aligned}
$$

respectively. Since $\left(\left(u^{-n}(e \gamma) u^{n}\right) \gamma^{m^{\prime}-m-1}\right) \beta^{n^{\prime}},\left(\left(u^{-n^{\prime}}\left(e^{\prime} \gamma\right) u^{n^{\prime}}\right) \gamma^{m-m^{\prime}-1}\right) \beta^{n} \in E(A)$, we clearly obtain $(m, n, e, n, m)\left(m^{\prime}, n^{\prime}, e^{\prime}, n^{\prime}, m^{\prime}\right)=\left(m^{\prime}, n^{\prime}, e^{\prime}, n^{\prime}, m^{\prime}\right)(m, n, e, n, m)$.

Hence, the result.

Competing interests

The authors declare that they have no competing interests.

Authors' contributions

All authors completed the paper together. All authors read and approved the final manuscript.

\section{Author details}

'Department of Mathematics, Kamil Özdag Science Faculty, Karamanoglu Mehmetbey University, Yunus Emre Campus, Karaman, 70100, Turkey. ${ }^{2}$ Department of Mathematics, Faculty of Science, Selçuk University, Campus, Konya, 42075, Turkey. ${ }^{3}$ Institute of Mathematics, Potsdam University, Potsdam, 14469, Germany. ${ }^{4}$ Department of Mathematics, Faculty of Arts and Science, Uludag University, Gorukle Campus, Bursa, 16059, Turkey.

\section{Acknowledgements}

Dedicated to Professor Hari M Srivastava.

The second and fourth authors are partially supported by Research Project Offices (BAP) of Selcuk (with Project No.

13701071) and Uludag (with Project No. 2012-15 and 2012-19) Universities, respectively. 


\section{References}

1. Howie, JM, Ruskuc, N: Constructions and presentations for monoids. Commun. Algebra 22(15), 6209-6224 (1994)

2. Bruck, RH: A Survey of Binary Systems. Ergebnisse der Mathematik, Neue Folge, vol. 20. Springer, Berlin (1958)

3. Munn, W: On simple inverse semigroups. Semigroup Forum 1, 63-74 (1970)

4. Reilly, NR: Bisimple w-semigroups. Proc. Glasg. Math. Assoc. 7, 160-167 (1966)

5. Kochin, BP: The structure of inverse ideal-simple $w$-semigroups. Vestn. Leningr. Univ. 23(7), 41-50 (1968)

6. Munn, W: Regular w-semigroups. Glasg. Math. J. 9, 46-66 (1968)

7. Asibong-lbe, U: *-Bisimple type A w-semigroups-I. Semigroup Forum 31, 99-117 (1985)

8. Shung, Y, Wang, LM: *-Bisimple type A $w^{2}$-semigroups as generalized Bruck-Reilly *-extensions. Southeast Asian Bull. Math. 32, 343-361 (2008)

9. Kocapinar, C, Karpuz, EG, Ateş, F, Çevik, AS: Gröbner-Shirshov bases of the generalized Bruck-Reilly *-extension. Algebra Colloq. 19(1), 813-820 (2012)

10. Nico, WR: On the regularity of semidirect products. J. Algebra 80, 29-36 (1983)

11. Zhang, $Y, L i, S$, Wang, D: Semidirect products and wreath products of strongly $\pi$-inverse monoids. Georgian Math. J. 3(3), 293-300 (1996)

12. Ateş, F: Some new monoid and group constructions under semidirect products. Ars Comb. 91, 203-218 (2009)

13. Karpuz, EG, Çevik, AS: A new example of strongly $\pi$-inverse monoids. Hacet. J. Math. Stat. 40(3), 461-468 (2011)

14. Clifford, AH, Preston, GB: The Algebraic Theory of Semigroups, vol. I. Mathematical Surveys, vol. 7. Am. Math. Soc., Providence (1964)

15. Clifford, AH, Preston, GB: The Algebraic Theory of Semigroups, vol. II. Mathematical Surveys, vol. 7. Am. Math. Soc., Providence (1967)

16. Howie, JM: Fundamentals of Semigroup Theory. Clarendon Press, New York (1995)

17. Bijev, G, Todorov, K: Coregular semigroups. In: Notes on Semigroups VI, pp. 1-11. Karl Marx Univ. Econom., Budapest (1980-1984)

doi:10.1186/1687-1812-2013-78

Cite this article as: Guzel Karpuz et al.: Some fixed-point results on (generalized) Bruck-Reilly $*$-extensions of monoids. Fixed Point Theory and Applications 2013 2013:78.

\section{Submit your manuscript to a SpringerOpen ${ }^{\ominus}$ journal and benefit from:}

- Convenient online submission

- Rigorous peer review

- Immediate publication on acceptance

- Open access: articles freely available online

- High visibility within the field

- Retaining the copyright to your article 